\title{
Les termites (Termitoidae Latreille 1802) ravageurs du manioc (Manihot esculenta Crantz 1766) dans la zone de Tivaouane (Sénégal)
}

\author{
Arfang Mafoudji SONKO ${ }^{1,3 *}$, Dienaba SALL ${ }^{2}$ et Abdoulaye Baïla NDIAYE ${ }^{1}$ \\ ${ }^{I}$ Université Cheikh Anta Diop (UCAD), Institut Fondamental d'Afrique Noire Cheikh A. Diop (IFAN Ch. Anta \\ Diop), Laboratoire de Zoologie des Invertébrés Terrestres, B. P. 206, Dakar, Sénégal. \\ ${ }^{2}$ Institut Sénégalais de Recherches Agricoles (ISRA), Centre de Recherches Agricoles de Saint-Louis (CRA), \\ Laboratoire d'Entomologie, BP, 3120 Dakar, Sénégal. \\ ${ }^{3}$ Université Cheikh Anta Diop (UCAD), Faculté des Sciences et Techniques, Département de Biologie \\ Végétale, 5005 Dakar-Fann, Sénégal. \\ *Auteur correspondant ; E-mail: arfang89@gmail.com; Tel: (+221) 772384455
}

\section{RÉSUMÉ}

Au Sénégal, le manioc, cultivé pour la consommation ou pour l'industrie, a des rendements faibles $(7,5$ t/ha en moyenne pour un potentiel de $40 \mathrm{t} / \mathrm{ha}$ ) à cause d'un certain nombre de contraintes dont les ravageurs. Les termites sont cités parmi les plus importants de ces ravageurs. Ainsi, il est mené cette étude qui a pour but de caractériser les attaques du manioc par les termites à Tivaouane (Sénégal). L'inventaire des espèces et l'évaluation de l'incidence des attaques ont été réalisés dans 5 sites. L'unité d'échantillonnage est une parcelle de 1 ha dans laquelle 100 plants, au minimum, sont observés. Cinq espèces de termites réparties entre champignonnistes (Odontotermes erraticus, Microtermes lepidus et Macrotermes subhyalinus) et lignivores (Psammotermes hybostoma et Microcerotermes sp.) ont été trouvées sur le manioc. M. lepidus, présente dans toutes les parcelles, est l'espèce dominante dans les attaques du manioc avec $56 \%$ de fréquence. Toutes parcelles confondues, l'incidence des attaques est de 37,5\% et l'indice de sévérité des attaques $48 \%$. Ainsi, dans le département de Tivaouane, il serait bon de tenir compte de M. lepidus dans l'itinéraire technique du manioc.

(C) 2019 International Formulae Group. All rights reserved.

Mots clés : Termites, attaques, manioc, Tivaouane, Sénégal.

\section{Termite (Termitoidae Latreille 1802) pest of cassava (Manihot esculenta Crantz 1766) in Tivaouane area (Senegal)}

\begin{abstract}
In Senegal, cassava, grown for food or for industry, has low yields $(7.5 \mathrm{t} / \mathrm{ha}$ on average for a potential of $40 \mathrm{t} / \mathrm{ha}$ ) due to some constraints including pests. Termites are supposed to be among the most important of those pests. So, this study aims to survey the attack of termite on cassava at Tivaouane (Senegal). The sampling of termite and the assessment of the incidence of their attacks were carried out in 5 sites. The sampling unit was a plot of 1 ha in which at least 100 plants were observed. Five species of termites that belong to fungus growing termites (Odontotermes erraticus, Microtermes lepidus and Macrotermes subhyalinus) and xylophagous (Psammotermes hybostoma and Microcerotermes sp.) have been found on cassava. M. lepidus,
\end{abstract}


present in all plots, is the dominant species in the attack of cassava with $56 \%$ frequency. All plots gathered, the incidence of attacks on cassava is $37.5 \%$ and the attack severity index $48 \%$. In Tivaouane department, the controle of M. lepidus should be integrated in the technology package of casssava production.

(C) 2019 International Formulae Group. All rights reserved.

Keywords: Termites, attacks, cassava, Tivaouane, Senegal.

\section{INTRODUCTION}

Le manioc, Manihot esculenta Crantz 1766, classé $4^{\text {ème }}$ production végétale au monde après le riz, le blé et le maïs, reste un aliment important pour plus d'un milliard de personnes, particulièrement en Afrique de l'Ouest où il est considéré comme une «denrée-sécurité-alimentaire » (SCA, 2013). Les tubercules de manioc sont consommées sous plusieurs formes; les feuilles, utilisées comme légume ou condiment, servent également à nourrir le bétail (Cacai et al., 2012 ; Diallo et al., 2013). Plus des deux tiers de la production de manioc sont destinés, sous diverses formes, à l'industrie agroalimentaire, à l'industrie textile, à la fabrication de colle, d'alcool, de papier, d'amidon, etc... (Agre et al., 2015 ; Kouakou et al., 2015).

Dans le monde, en 2014, la production de racines et de tubercules a atteint 845 millions de tonnes en matière fraiche dont $32 \%$ représentés par le manioc (Vernier et al., 2018). L'Afrique, avec $57 \%$ de la production mondiale, est le leader de la filière manioc (FAOSTAT, 2014). Le Nigeria, avec 54831600 t/an, est le plus grand producteur mondial de manioc (FAOSTAT, 2014).

$\mathrm{Au}$ Sénégal, le manioc est l'une des plantes à tubercules les plus cultivées, et ce bien avant l'indépendance (SCA, 2013). La production a connu un succès de 1960 à 1975 avec des volumes parfois supérieurs à 250000 tonnes selon DAPSA (2013). Le potentiel de production du pays, estimé à 1000000 de tonnes par an (SCA, 2013), est essentiellement fondé sur l'augmentation des surfaces cultivées. Ainsi, selon la DAPSA (2017), de 2013 à 2015 les superficies de manioc sont passées de 20868 hectares à 30813 hectares alors que le rendement moyen, 6 à 7,58 t/ha, est resté le même. Le département de Tivaouane, qui produit 55\% de l'offre nationale (SCA, 2013), enregistre les rendements les plus faibles (7,5 t/ha en moyenne) par rapport aux autres départements de production de manioc (DAPSA, 2017): Nioro (22,5 t/ha), Mbour (9 t/ha), Thiès ( 9 t/ha) et Vélingara ( $8 \mathrm{t} / \mathrm{ha})$. Cette faiblesse des rendements serait liée aux contraintes phytosanitaires dont l'action des termites ravageurs. Les termites qui s'attaquent aux boutures, aux tiges, aux racines et aux tubercules, provoqueraient des baisses de rendement au champ (CDDR/SAILD, 2013).

En Afrique de 1'Ouest, les attaques de termites sur les plantes sont bien documentées. Au Togo, Kotoklo et al. (2010 ; 2011) ont relevé des attaques de termites sur des arbres du campus de l'Université de Lomé et dans des plantations de canne à sucre. En Côte d'Ivoire, d'après Akpesse et al. (2008), les dommages causés par les termites sur le maïs et sur le riz au champ étaient respectivement de 60,8 et $61,9 \%$. Au Sénégal, différentes études ont montré l'importance des attaques des termites sur les cultures (Ndiaye et Han, 2002; 2006 ; 2007 ; Sonko, 2015, Sané et al., 2016).

Concernant le manioc, les attaques de termites sont relativement peu documentées. En Afrique, Pearce (2006) et Rouland-Lefèvre (2011) rapportent des cas de dommages causés par les termites. Pour le Sénégal, à notre connaissance il n'existe que deux références (Faye et al., 2014 ; Faye 2016) portant sur la sensibilité de différentes variétés de manioc aux attaques de termites en milieu semi-contrôlé. Ainsi, dans le cadre d'une contribution à l'amélioration de la productivité du manioc au Sénégal, la présente étude a été menée dans le département de Tivaouane (région de Thiès, Sénégal) pour i) connaitre les espèces de termites qui attaquent 
le manioc et ii) évaluer l'incidence et la sévérité de leurs attaques.

\section{MATERIEL ET METHODES Sites d'étude}

L'étude a été réalisée dans les localités de Koul, Meouane, Pire, Taïba Ndiaye et Tivaouane, situées dans la région de Thiès et dans le département de Tivaouane (Figure 1).

Le climat, de type sahélo-soudanien, est caractérisé par une courte saison des pluies (juillet-octobre) et une longue saison sèche (octobre-juin). Les températures les plus basses $\left(16^{\circ} \mathrm{C}\right)$ sont enregistrées entre décembre et février, et les plus fortes $\left(35^{\circ} \mathrm{C}\right)$ de mars à octobre (Le Moigne et al., 2012). Le cumul annuel de pluie est compris entre 400 et $600 \mathrm{~mm}$ (ANSD/SRSD, 2015).

La végétation est une savane arbustive dégradée parsemée de Acacia seyal Del, de Adansonia digitata $\mathrm{L}$, de Acacia albida Del et de Borassus aethiopum Mart. Un tapis herbacé, dominé par des graminées, se développe entre les ligneux pendant la saison des pluies. Les sols sont i) ferrugineux tropicaux lessivés à texture sableuse, ii) ferrugineux tropicaux à texture argilo-sableux, iii) ferrugineux tropicaux lessivés à texture argilo-humique ou iv) hydromorphes à texture humique au niveau des bas-fonds (ANSD/SRSD, 2015).

\section{Échantillonnage des termites}

L'unité d'échantillonnage est une parcelle de 1 ha. Le nombre de parcelles échantillonnées est de 5 à Koul, 3 à Meouane, 3 à Pire, 4 Taïba Ndiaye et 3 Tivaouane. Dans chaque parcelle, un minimum de 100 plants de manioc sont observés en parcourant les lignes de manière à couvrir toute l'unité d'échantillonnage. L'âge de la plantation est relevé. Les observations portent sur l'état du plant, les signes d'attaques, le niveau des dégâts et sur la présence de termites. Les pieds morts ou mourants sont enlevés pour une observation de la partie en terre. Les termites présents sont récoltés et mis dans des tubes contenant de l'éthanol $70^{\circ}$ et étiquetés.

\section{Identification des spécimens}

En plus des spécimens de référence de la collection du Laboratoire de Zoologie des Invertébrés terrestres de l'Institut fondamental d'Afrique noire (IFAN), les travaux de Bouillon \& Mathot (1965), de Grassé (1944) et de Ndiaye (2014) ont été utilisés pour identifier les termites récoltés sur le manioc.

\section{Paramètres étudiés}

La diversité (S) des termites correspond aux espèces recensées sur le manioc.

La distribution des espèces est évaluée par leur constance (C) dans les parcelles. La constance d'une espèce, calculée selon la formule modifiée de Dajoz (1971), correspond au nombre de parcelles où l'espèce est trouvée sur le nombre total de parcelles échantillonnées.

$$
\mathrm{C}=\frac{\text { Nombre de parcelles où l'espèce est trouvée }}{\text { Nombre de parcelles échantillonnées }} 100
$$

En suivant les classes définies par Dajoz (1971), les espèces seront classées en :

- espèces constantes pour des valeurs de $\mathrm{C} \geq 50 \%$ ) ;

- espèces accessoires pour $25 \leq \mathrm{C}<$ $50 \%)$;

- espèces accidentelles pour $\mathrm{C}<25 \%$ ).

\section{La fréquence des espèces sur le manioc}

La fréquence d'une espèce $(F)$, adaptée de la formule de Dajoz (1971) pour le cas des termites, est définie ici comme le nombre d'échantillons contenant l'espèce sur le nombre total d'échantillons de termites prélevés sur le manioc. L'échantillon correspond aux termites prélevés sur le même plant de manioc et conservés dans le même tube.

$$
\mathrm{F}=\frac{\text { Nombre d'échantillons contenant l'espèce }}{\text { Nombre total d'échantillons }} 100
$$

Une espèce est dite :

- $\quad$ rare lorsque $\mathrm{F}<5 \%$;

- assez fréquente pour $5 \leq \mathrm{F}<25 \%$;

- fréquente pour $25 \leq \mathrm{F}<50 \%$;

- très fréquente pour des valeurs de $\mathrm{F} \geq$ $50 \%$. 


\section{L'incidence des attaques}

L'incidence (I) des attaques correspond au nombre de pieds de manioc attaqués par rapport au nombre total de pieds observés. Il est calculé par la formule :

\section{$\mathrm{I}=\frac{\text { Nombre de plants attaqués }}{\text { Nombre total de plants observés }} 100$}

\section{L'indice de sévérité des attaques}

Les dégâts des termites sont classés selon une échelle de cotations allant de 1 à 5 (Tableau 1). La sévérité des attaques, évaluée par l'indice de sévérité (Is), permet d'apprécier le niveau des dégâts des termites. Il est calculé par la formule suivante :
Is $=\frac{\sum X i N i}{N . Z} 100$

Xi : sévérité i (de 1 à 5) des attaques sur le manioc

$\mathrm{Ni}$ : nombre de pieds de manioc ayant subi une attaque de sévérité $\mathrm{i}$

$\mathrm{Z}$ : échelle de sévérité la plus élevée.

\section{Analyses statistiques}

Une analyse de variance (ANOVA) au seuil de 5\% et le test de Fisher réalisés avec le logiciel XLSTAT (version 2014) ont été utilisés pour les tests de comparaison des moyennes.

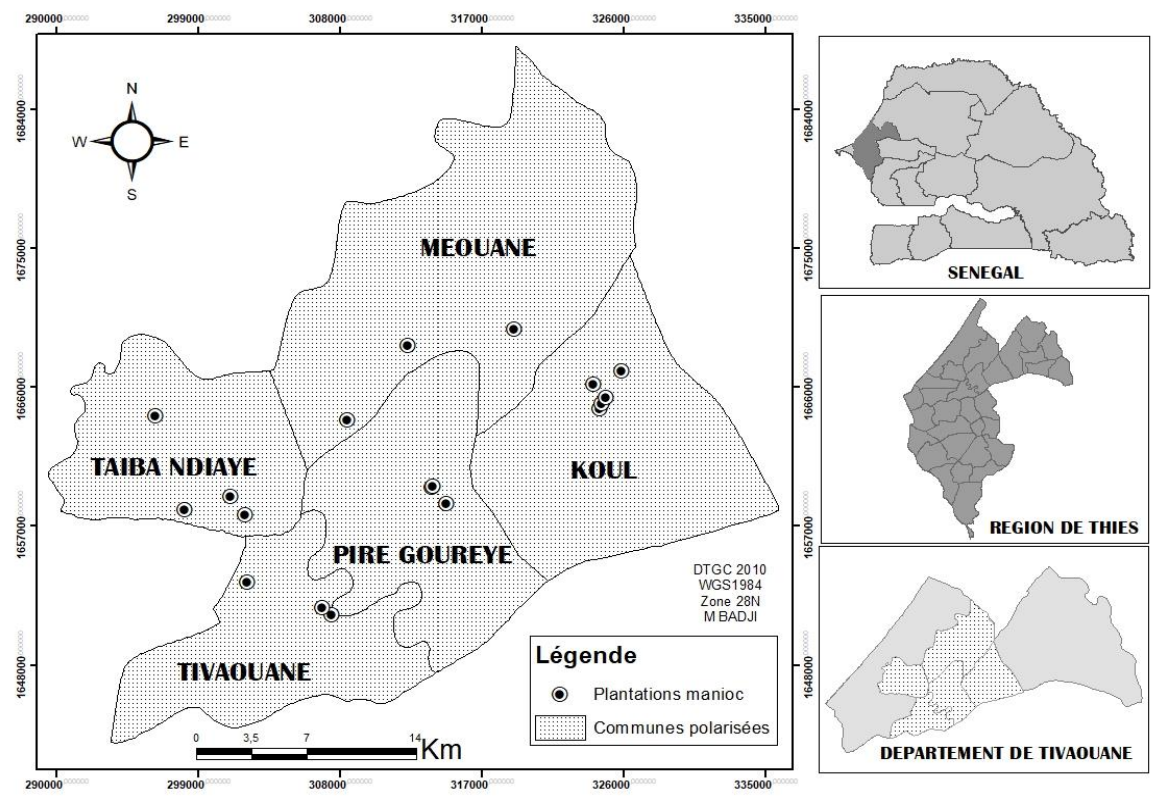

Figure 1: Localisation des sites et des parcelles d'échantillonnage de termites sur le manioc à Tivaouane.

Tableau 1 : Échelle de cotation des attaques de termites sur le manioc.

\begin{tabular}{ll}
\hline Niveau & Description \\
\hline $\mathbf{1}$ & Pas de trace d'activité de termites sur les parties visibles du pied observé \\
\hline $\mathbf{2}$ & $\begin{array}{l}\text { Présence de galeries et/ou placages externes de termites sur le pied mais sans } \\
\text { dommage }(25 \%)\end{array}$ \\
\hline $\mathbf{3}$ & $\begin{array}{l}\text { Galeries et/ou placages externes et/ou internes avec des dommages sur l'écorce et } \\
\text { le cour de la tige (50\%) }\end{array}$ \\
\hline $\mathbf{4}$ & Tige en partie détruite par les attaques de termites (75\%) \\
\hline $\mathbf{5}$ & Pied mourant ou mort (100\%) \\
\hline
\end{tabular}




\section{RESULTATS}

\section{Diversité des espèces}

Cinq espèces de termites qui s'attaquent au manioc ont été répertoriées (Tableau 2). Elles se répartissent entre les familles Rhinotermitidae et Termitidae. Macrotermes subhyalinus n'a été trouvé sur le manioc qu'une seule à Taïba Ndiaye sur des boutures non encore plantées. Cependant, des placages caractéristiques de cette espèce trouvés sur des pieds rongés indiquent clairement qu'elle s'attaque au manioc.

$\mathrm{Au}$ niveau fonctionnel, les termites rencontrés se répartissent entre lignivores et champignonnistes. Les lignivores sont : Psammotermes hybostoma (Figure 2) et Microcerotermes sp. (Figure 3). Les champignonnistes sont constitués de: Macrotermes subhyalinus (Figure 4), Microtermes lepidus (Figure 5) et Odontotermes erraticus (Figure 6).

Les attaques des termites peuvent se faire de l'extérieur sous des placages de terre (Figure 7), ou de l'intérieur avec remplissage ou non de terre des parties rongées (Figure 8). Elles peuvent endommager (Figure 9) et même tuer le plant attaqué (Figure 10).

\section{Constance des espèces}

Les résultats ont révélé la présence de deux espèces constantes appartenant au groupe des champignonnistes. Il s'agit de Microtermes lepidus et de Odontotermes erraticus présents respectivement dans $100 \%$ et $66,7 \%$ des parcelles. Les deux autres espèces Psammotermes hybostoma $(\mathrm{C}=44$, $4 \%)$ et Microcerotermes sp. $(\mathrm{C}=5,6 \%)$ sont respectivement accessoires et accidentelles.

\section{Fréquence des espèces de termites sur le manioc}

La fréquence des espèces tous sites confondus (Figure 11) montre que Microcerotermes sp. est rare $(\mathrm{F}=0,8 \%)$, Odontotermes erraticus assez fréquente $(\mathrm{F}=$ $12 \%)$, Psammotermes hybostoma fréquente (F $=31,2 \%$ ) et l'espèce Microtermes lepidus très fréquente $(\mathrm{F}=56 \%)$.

A Koul, par sa fréquence sur le manioc, Microtermes lepidus domine
Psammotermes hybostoma et Odontotermes erraticus (Figure 12).

À Meouane, Microtermes lepidus est très fréquente sur le manioc, Psammotermes hybostoma fréquente, Odontotermes erraticus assez fréquente dans les attaques et Microcerotermes sp. rare (Figure 13).

À Pire, sur le manioc, Psammotermes hybostoma est très fréquente, $M$. lepidus fréquente et Odontotermes erraticus assez fréquente (Figure 14).

À Taïba Ndiaye, la fréquence des espèces récoltées sur le manioc (Figure 15) montre une dominance de Microtermes lepidus $(\mathrm{F}=73,7 \%)$. Odontotermes erraticus est ici fréquente.

À de Tivaouane, Microtermes lepidus est très fréquente et Odontotermes erraticus assez fréquente (Figure 16).

\section{Incidence des attaques du manioc}

$\mathrm{Au}$ niveau des 18 parcelles échantillonnées, 3321 pieds de manioc ont été observés. Il a été relevé 1205 pieds manquants, soit $26,6 \%$ des boutures plantées au départ. L'incidence des attaques du manioc, tous sites confondus, est de $37,5 \%$. D'un site à l'autre une certaine variation est observée (Figure 17). L'incidence des attaques est plus élevée à Meouane $(58,1 \%)$ et à Pire $(55,8 \%)$. Elle n'est pas significativement différente entre les deux sites $(\mathrm{DDL}=4 ; \mathrm{F}=4,632 ; \mathrm{P}=0,88)$. L'incidence la plus faible est observée à Tivaouane $(26,6 \%)$ et Taïba Ndiaye (22\%). L'incidence des attaques entre ces deux sites n'est pas significativement différente (DDL $=$ 4; $\mathrm{F}=4,632 ; \mathrm{P}=0,6)$. L'incidence des attaques observée à Koul $(38,9 \%)$ ne montre de différence significative avec aucun des sites (Tableau 3). Concernant l'âge des plants de manioc, il est observé une certaine corrélation négative entre l'incidence des attaques et l'âge des parcelles (Figure 18). D'après la pente de la courbe, la corrélation est plus forte les premiers mois après la plantation du manioc. 
L'indice de sévérité des attaques de termites sur le manioc

L'indice de sévérité moyen des attaques de termites sur le manioc tous sites confondus est de $48 \%$. Il est variable selon les sites (Figure 19). Les indices de sévérité les plus faibles sont observés à Taïba Ndiaye
$(\mathrm{Is}=34 \%)$ et à Tivaouane (Is=36\%) et les plus élevés à Meouane (Is=58\%) et à Pire (Is=63\%). À Koul l'indice de sévérité des attaques est de $46 \%$. Il est également observé une corrélation négative entre l'âge des plants et la sévérité des attaques (Figure 20).

Tableau 2: Les espèces répertoriées.

\begin{tabular}{|c|c|c|}
\hline Famille & Sous-famille & Espèce \\
\hline $\begin{array}{l}\text { Rhinotermitidae } \\
\text { Light } 1921\end{array}$ & $\begin{array}{l}\text { Psammotermitinae Holmgren } \\
1911\end{array}$ & Psammotermes hybostoma Desneux 1902 \\
\hline \multirow{3}{*}{$\begin{array}{l}\text { Termitidae } \\
\text { Light } 1921\end{array}$} & \multirow{2}{*}{ Macrotermitinae Kemner 1934} & $\begin{array}{l}\text { Macrotermes subhyalinus (Rambur 1847) } \\
\text { Microtermes lepidus Sjöstedt } 1925\end{array}$ \\
\hline & & Odontotermes erraticus Grassé 1944 \\
\hline & Termitinae & Microcerotermes sp. \\
\hline
\end{tabular}

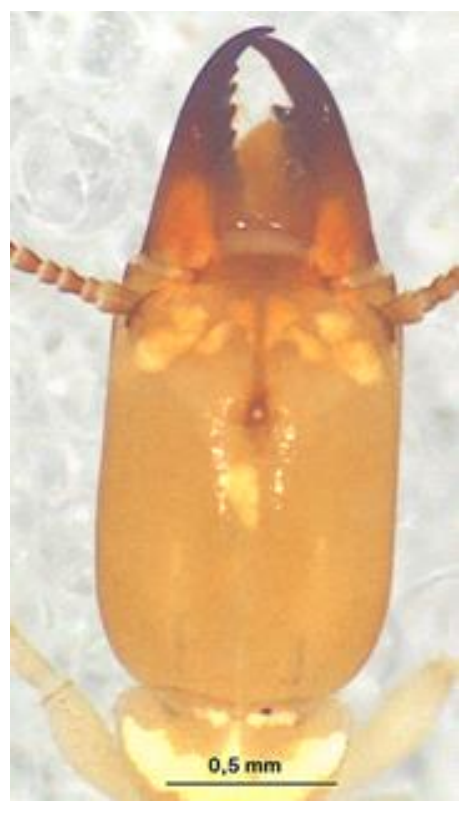

Figure 2: Tête du soldat moyen de Psammotermes hybostoma.

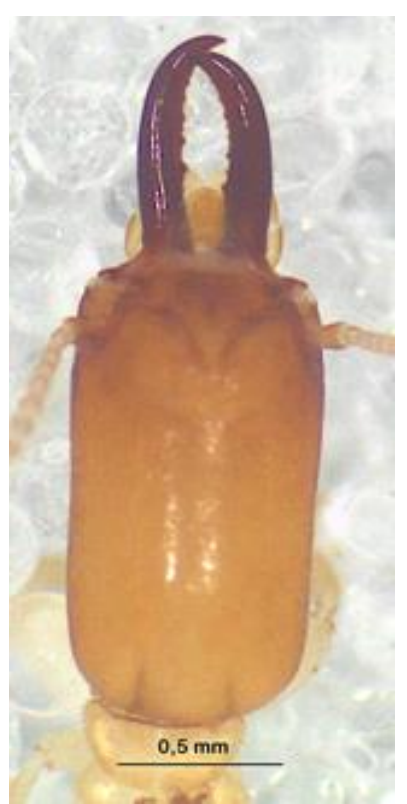

Figure 3 : Tête du soldat de Microcerotermes sp. 


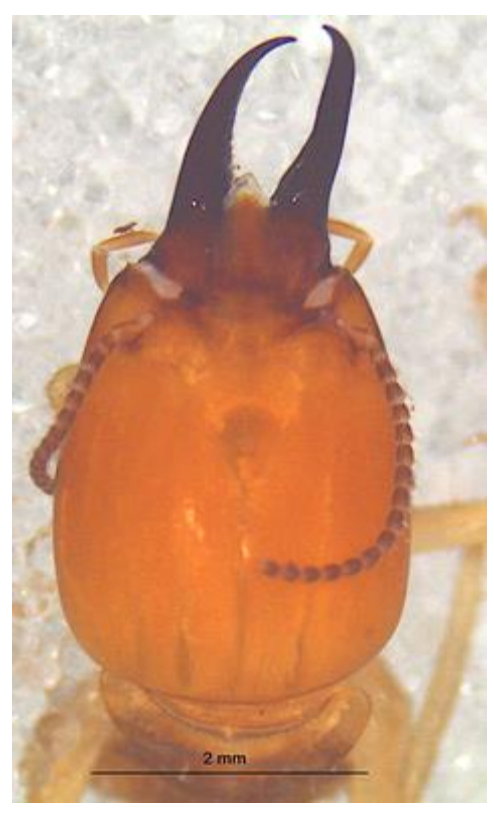

Figure 4: Tête du petit soldat de Macrotermes subhyalinus.

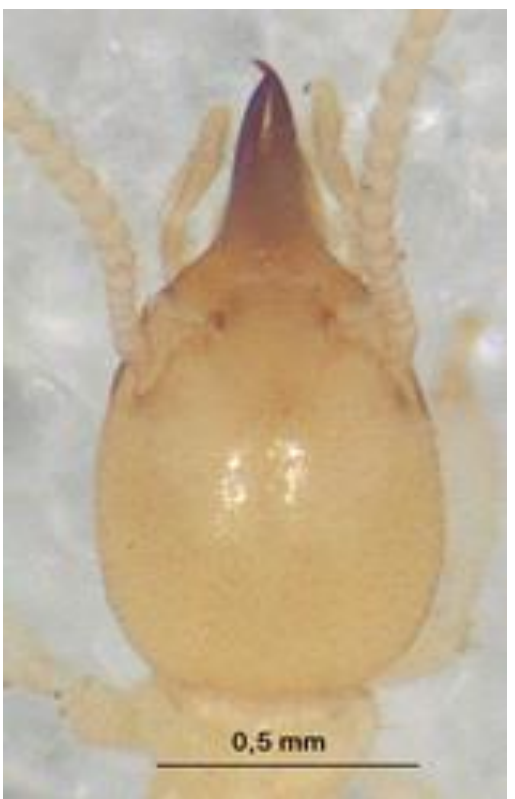

Figure 5 : Tête du soldat de Microtermes lepidus.

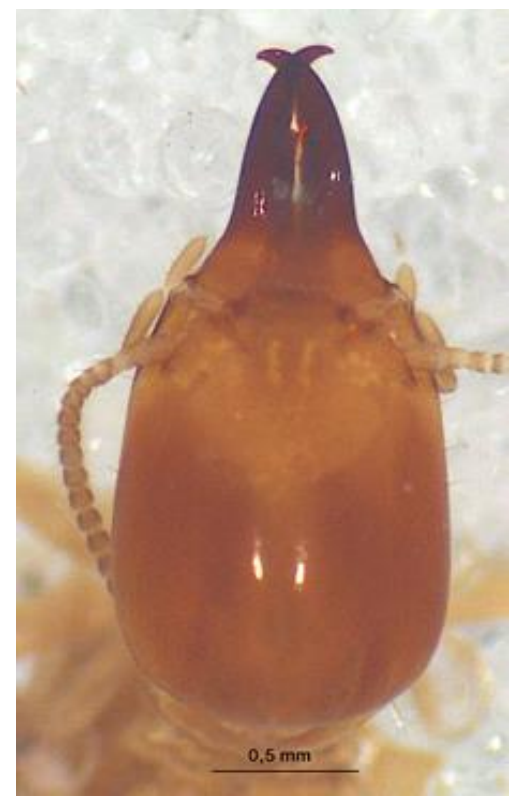

Figure 6 : Tête du soldat de Odontotermes erraticus Grassé. 

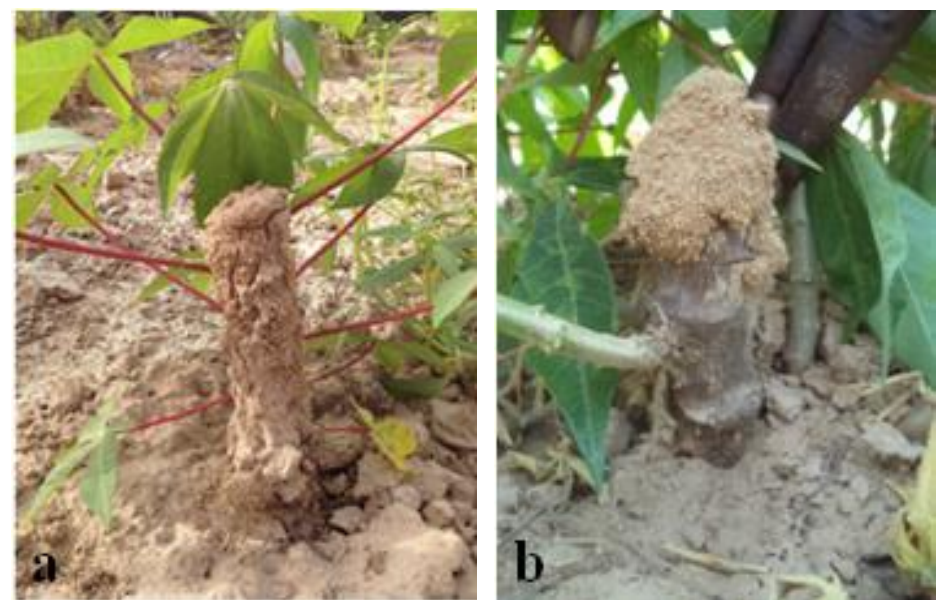

Figure 7: Placages de Odontotermes erraticus (a) et de Microtermes lepidus (b) sur du manioc.
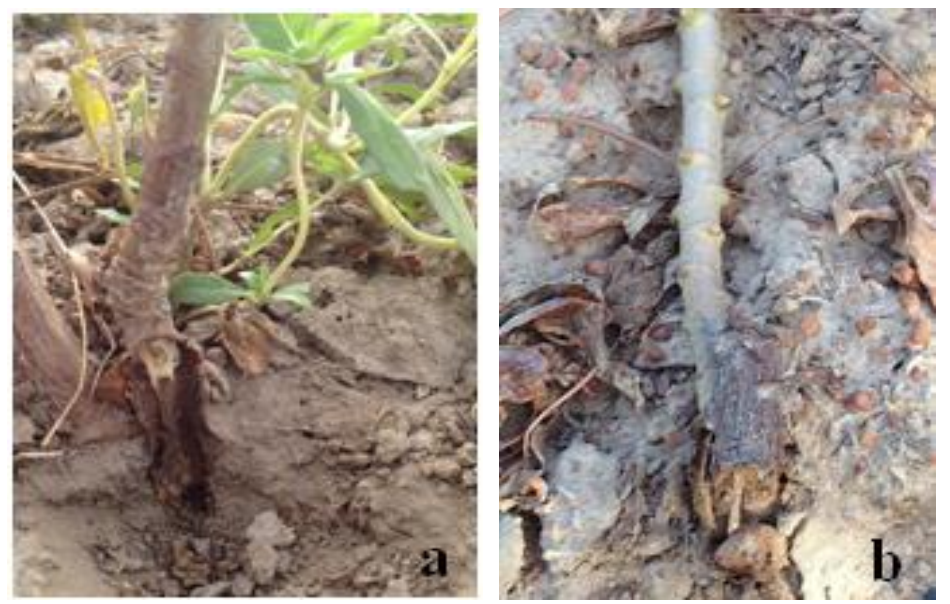

Figure 8: Attaques internes sans remplissage de terre (a) et avec remplissage de terre (b) de plants de manioc par Microtermes lepidus.
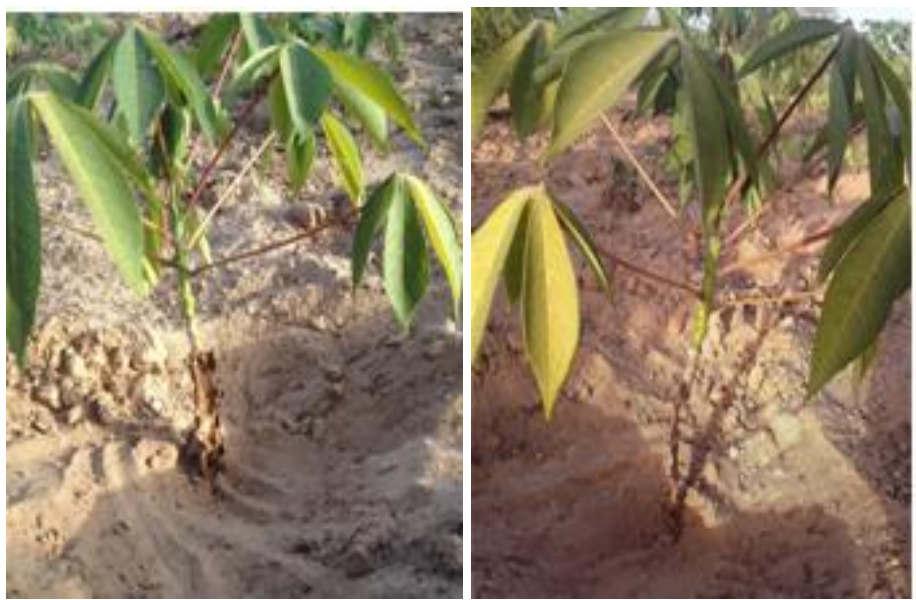

Figure 9 : Plant de manioc endommagé par des attaques de Microtermes lepidus. 


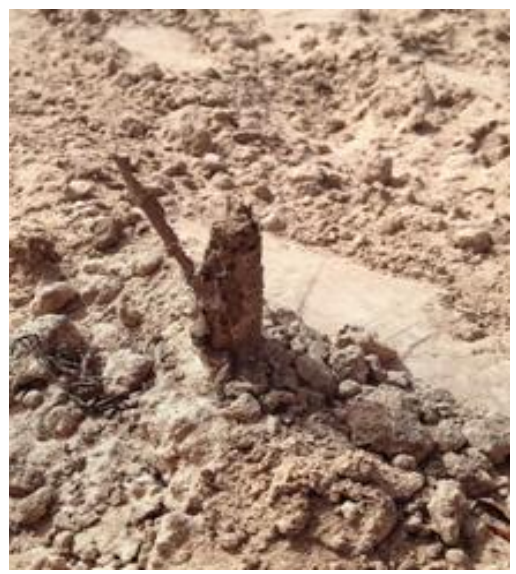

Figure 10 : Plant de manioc mort attaqué par Psammotermes hybostoma.

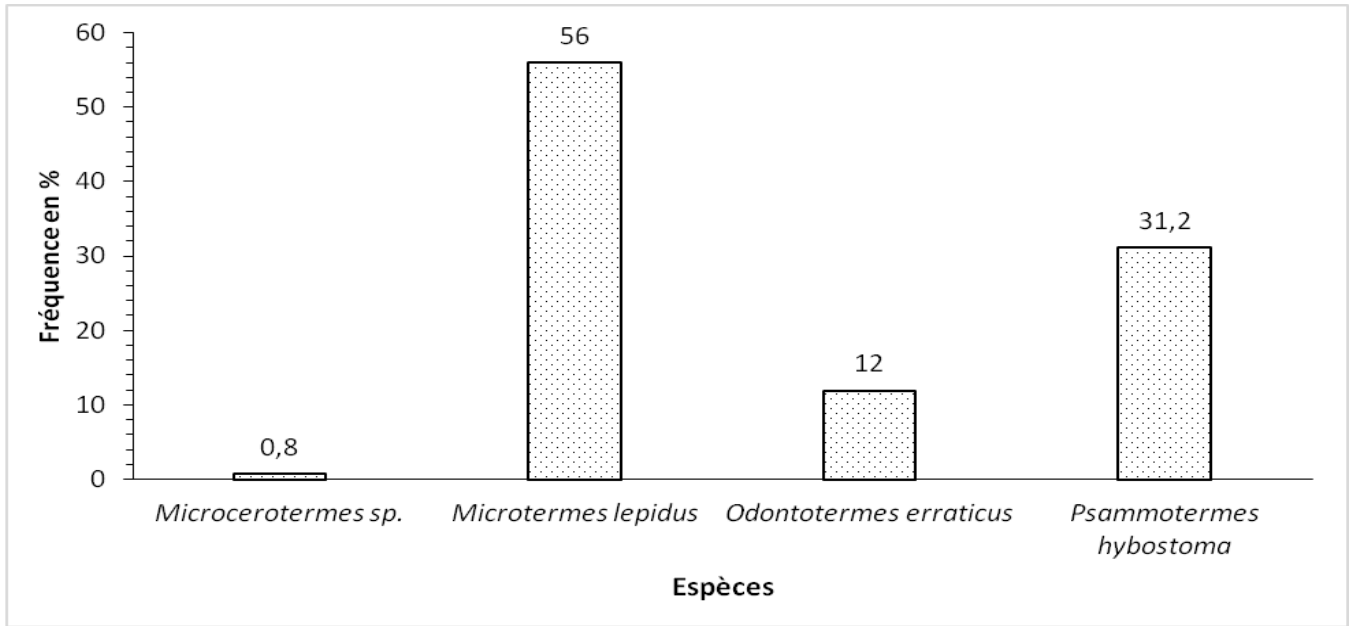

Figure 11 : Fréquence des termites sur le manioc tous sites confondus à Tivaouane.

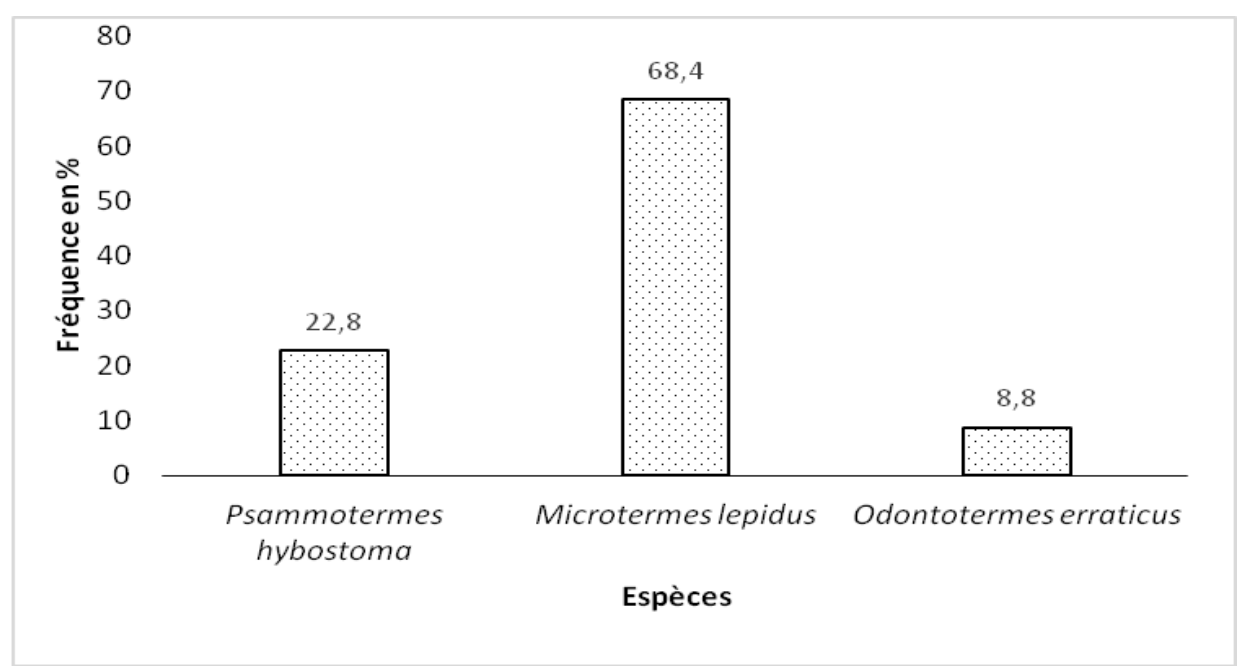

Figure 12 : Fréquence des termites sur le manioc à Koul (Tivaouane). 


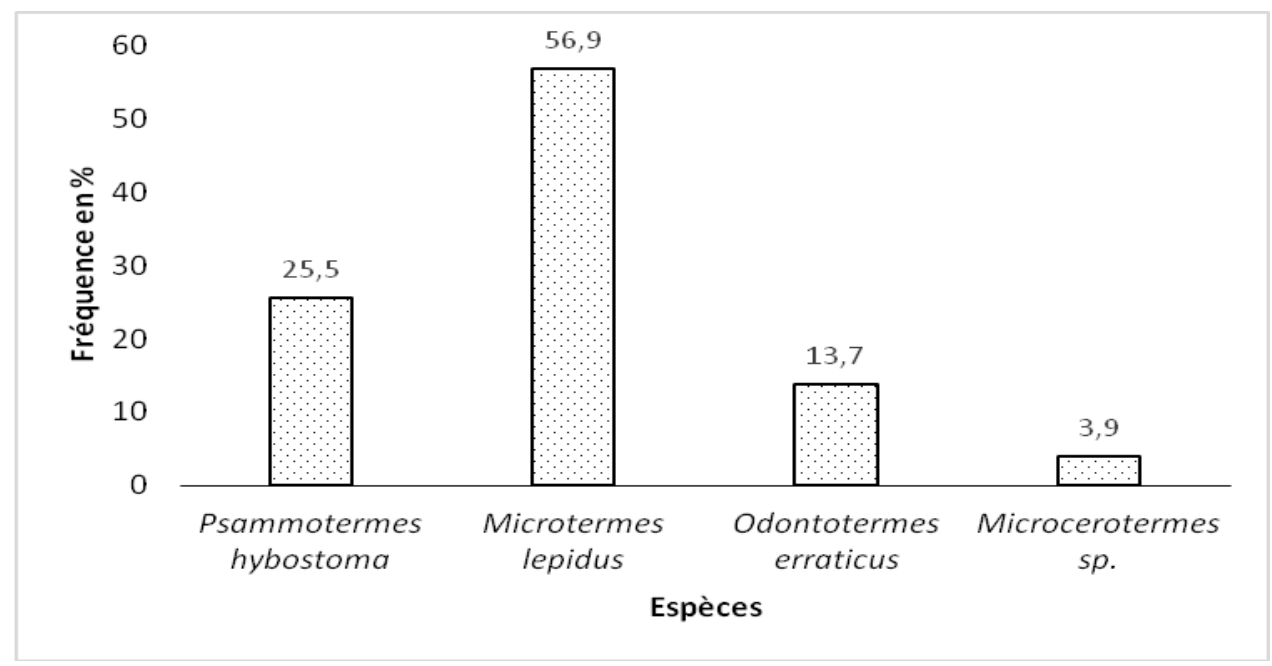

Figure 13 : Fréquence des termites sur le manioc à Meouane (Tivaouane).

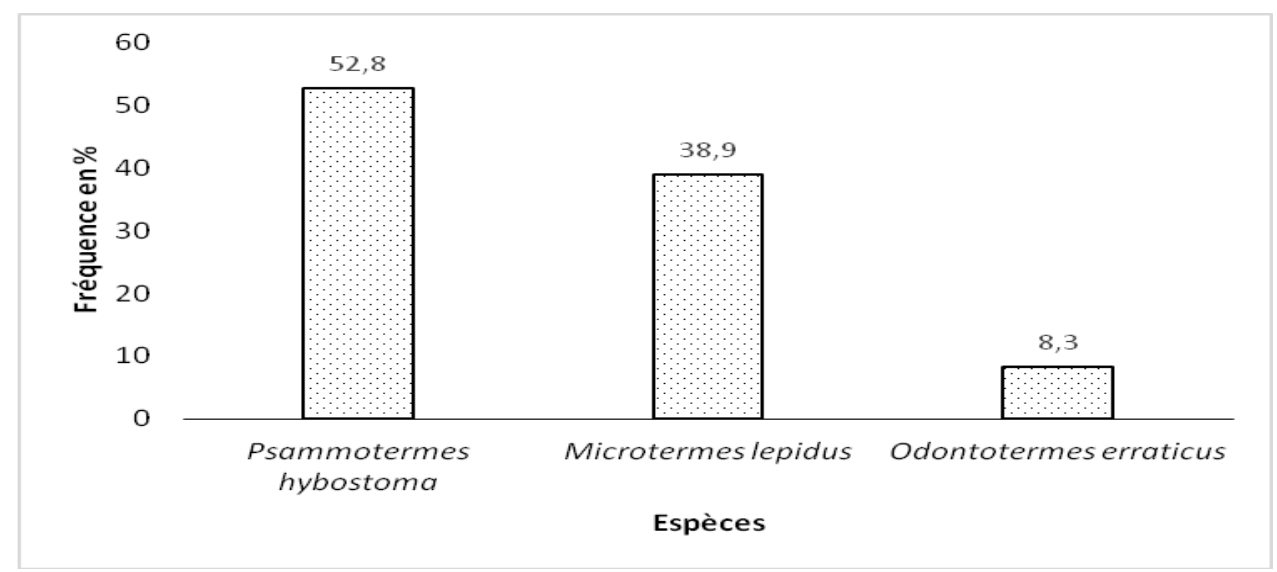

Figure 14 : Fréquence des termites sur le manioc à Pire (Tivaouane).

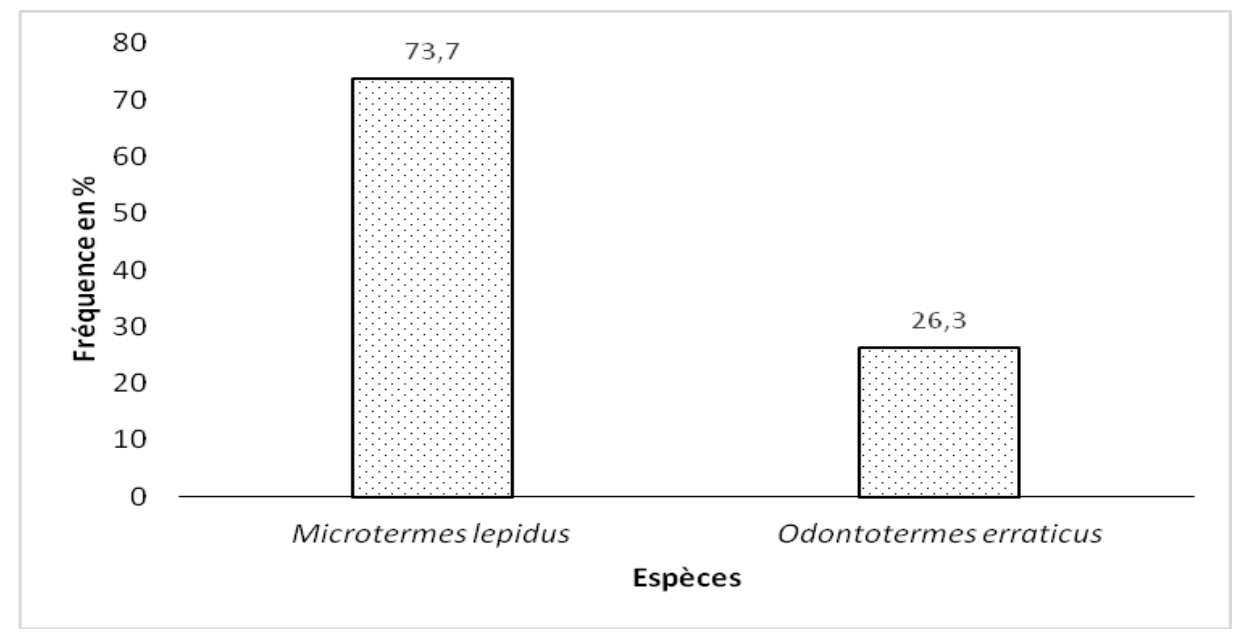

Figure 15 : Fréquence des termites sur le manioc à Taïba Ndiaye (Tivaouane). 


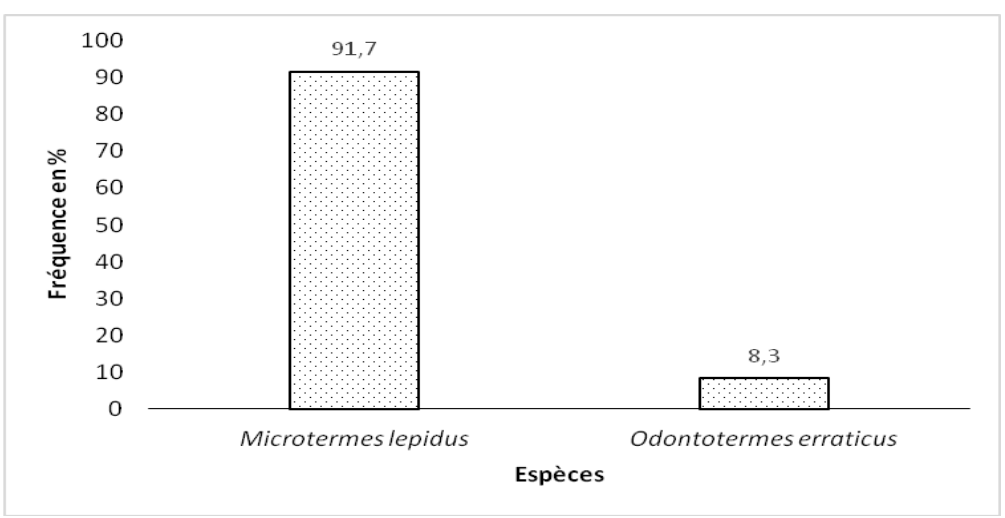

Figure 16: Fréquence des termites sur le manioc à Tivaouane (Tivaouane).

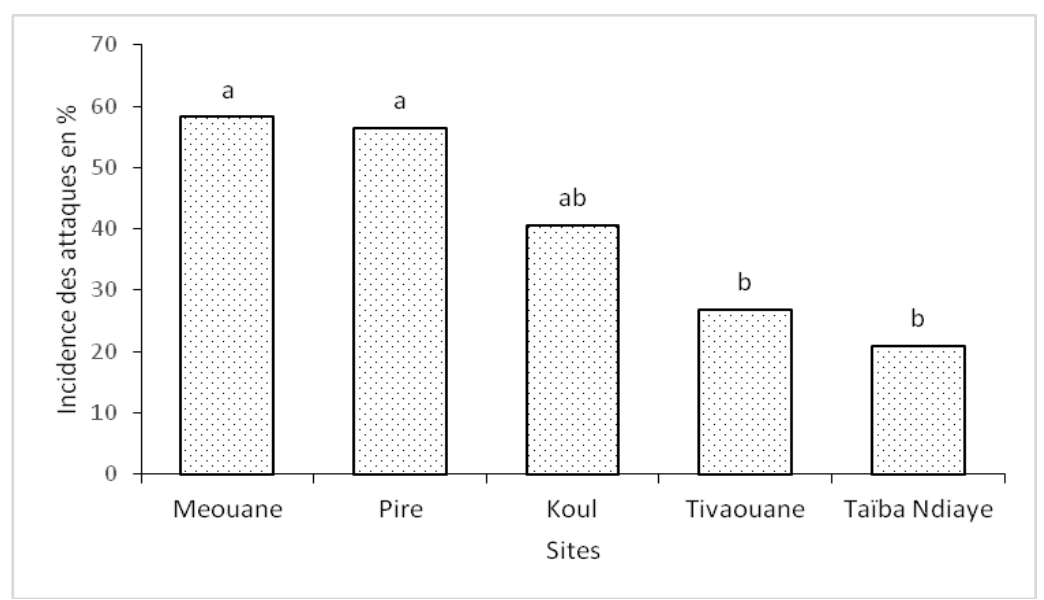

Figure 17: Incidence des attaques de termites sur le manioc dans les sites étudiés à Tivaouane.

Tableau 3 : Analyse des différences entre incidences des attaques des termites entre sites (Test de Fisher, intervalle de confiance à $95 \%, \mathrm{ddl}=4, \mathrm{~F}=4,6320$ ).

\begin{tabular}{lll}
\hline Sites comparés & $\mathbf{P}$ & Significatif \\
\hline Meouane vs Taïba Ndiaye & 0,0046 & Oui \\
\hline Meouane vs Tivaouane & 0,0184 & Oui \\
\hline Meouane vs Koul & 0,1133 & Non \\
\hline Meouane vs Pire & 0,8839 & Non \\
\hline Pire vs Taïba Ndiaye & 0,0063 & Oui \\
\hline Pire vs Tivaouane & 0,0244 & Oui \\
\hline Pire vs Koul & 0,1496 & Non \\
\hline Koul vs Taïba Ndiaye & 0,0623 & Non \\
\hline Koul vs Tivaouane & 0,2114 & Non \\
\hline Tivaouane vs Taïba Ndiaye & 0,6021 & Non \\
\hline
\end{tabular}




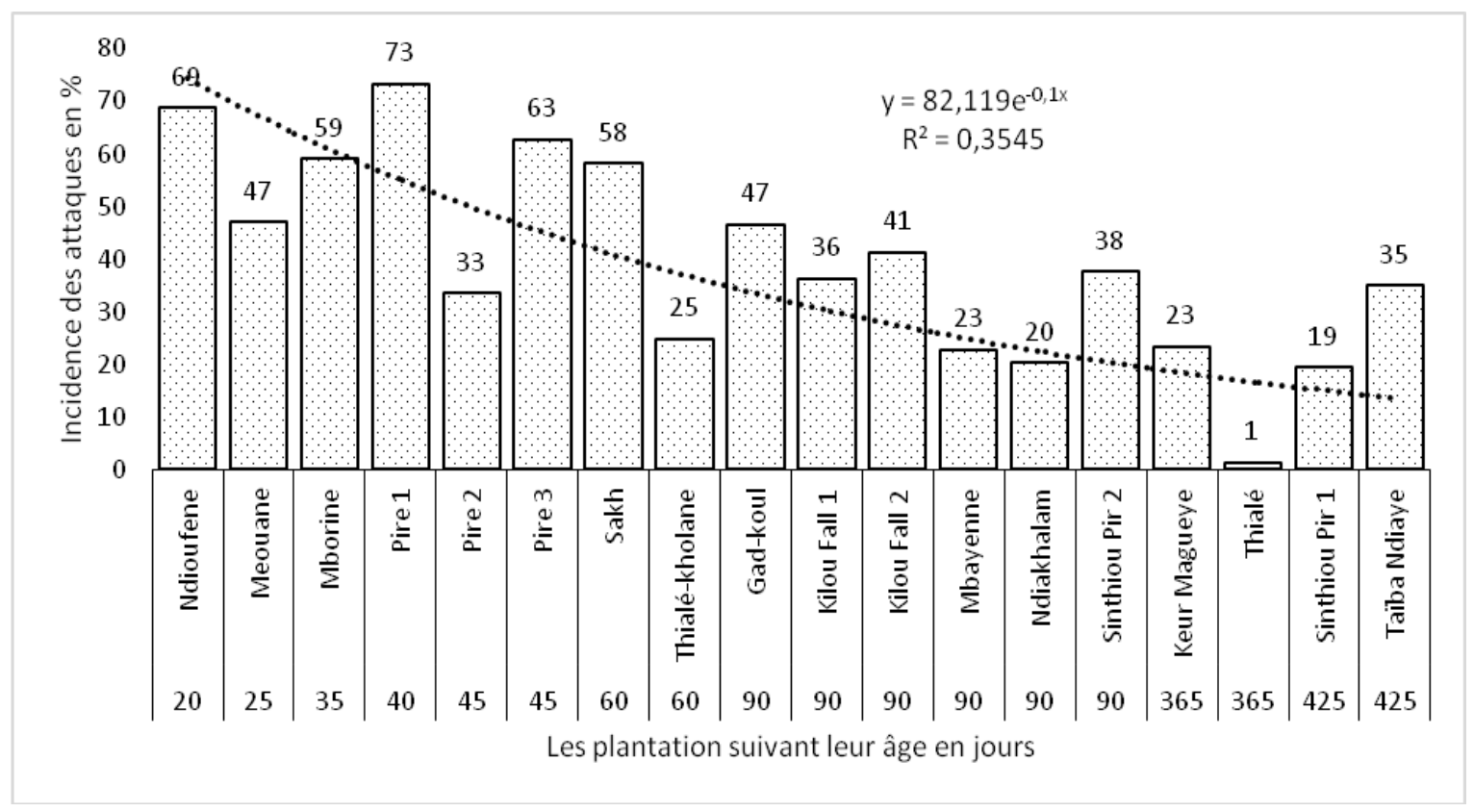

Figure 18: Incidence des attaques de termites sur le manioc en fonction de l'âge des plants à Tivaouane.

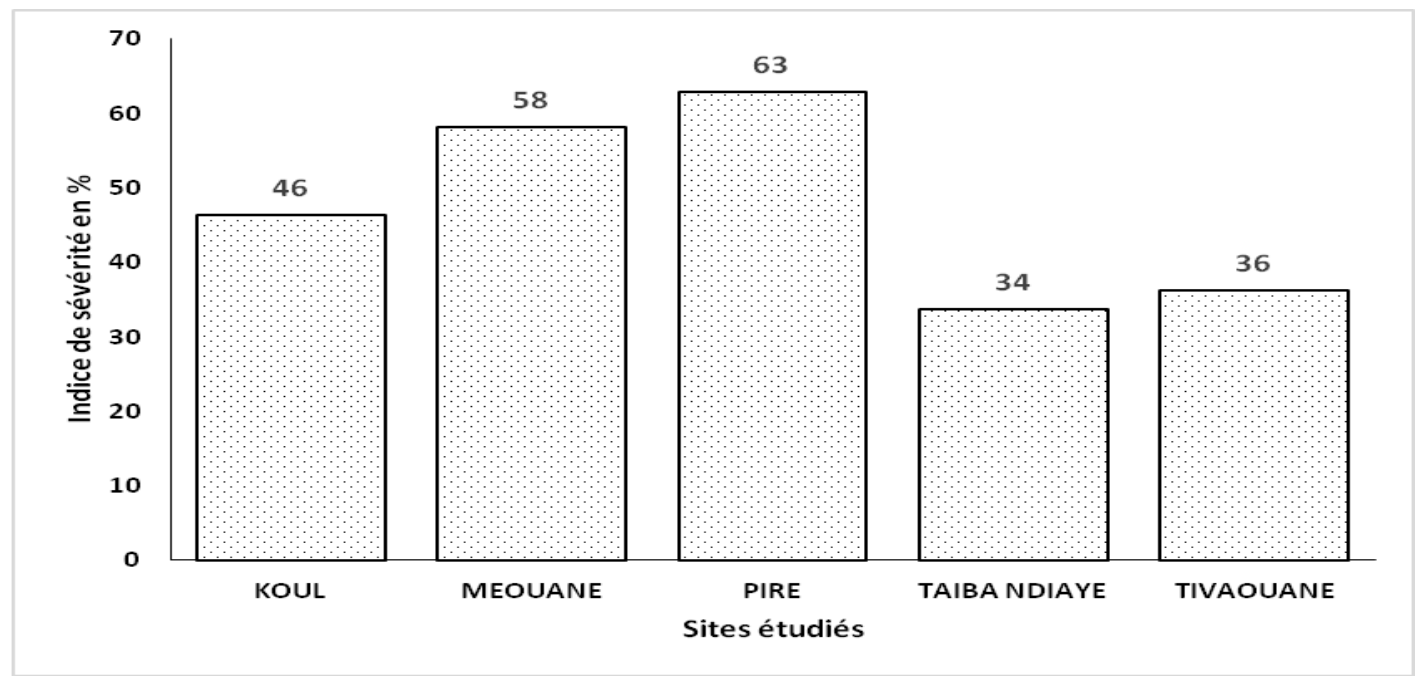

Figure 19 : Sévérité des attaques de termites sur le manioc dans les sites étudiés à Tivaouane. 


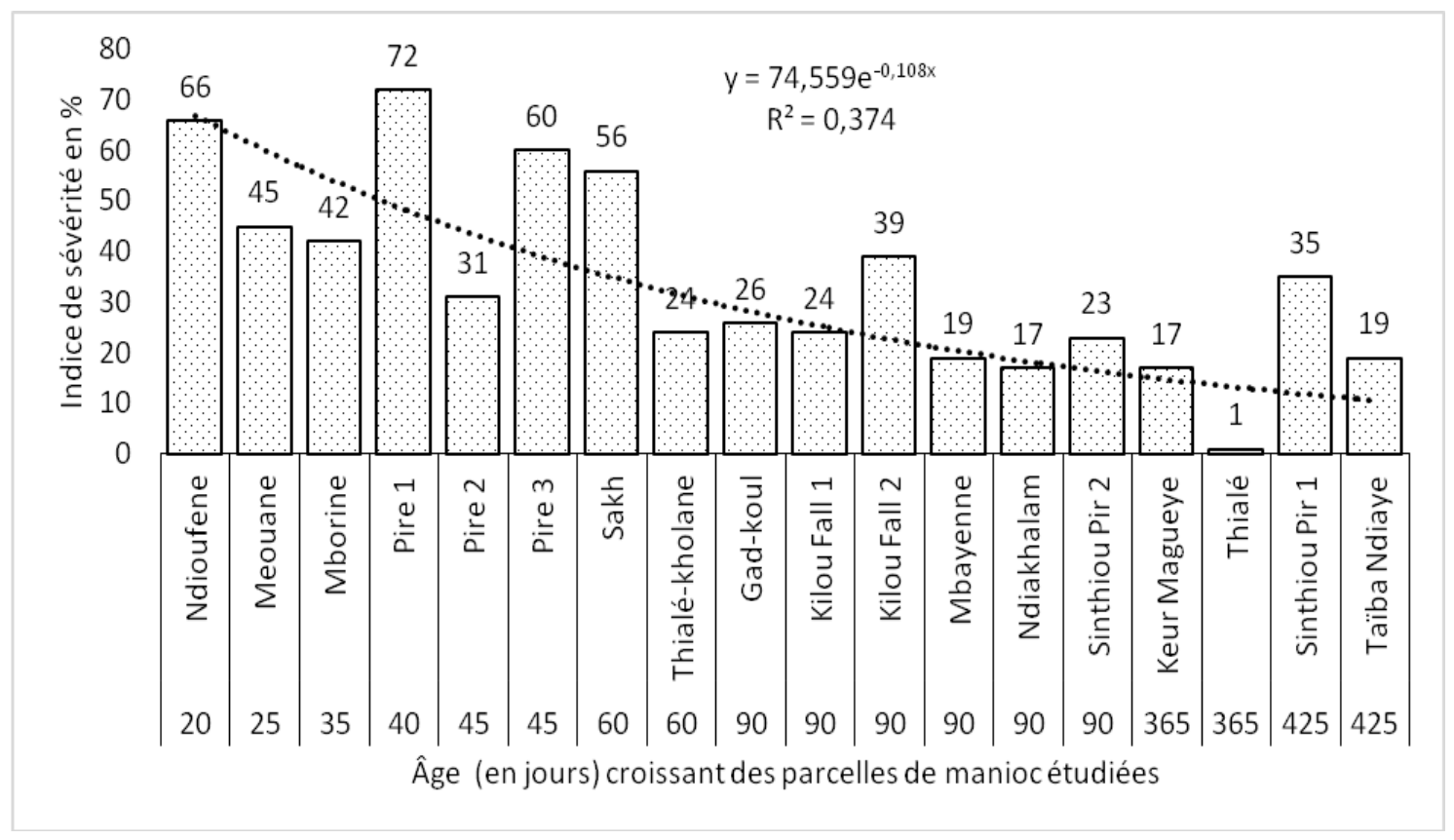

Figure 20 : Sévérité des attaques de termites sur le manioc selon l'âge des plants à Tivaouane.

\section{DISCUSSION}

L'étude a permis de recenser sur le manioc 5 espèces de termites: Psammotermes hybostoma, Microcerotermes sp., Microtermes lepidus, Macrotermes subhyalinus et Odontotermes erraticus. Faye (2016) avait recensé le même nombre d'espèces de termites dans des agrosystèmes manioc. Mais les listes des espèces diffèrent, au niveau qualitatif, par la présence d'Amitermes evuncifer et l'absence de Microcerotermes sp. chez Faye (2016). Ainsi, on aurait une liste de 6 espèces de termites qui attaquent le manioc dans le département de Tivaouane.

Les Macrotermitinae Macrotermes, Microtermes et Odontotermes sont considérés par Pearce (2006) comme économiquement les plus importants genres qui s'attaquent aux cultures en Afrique. Au Tchad, Abderahim et al. (2018) signalent Odontotermes sp. aff. erraticus parmi les arthropodes ravageurs du manioc.

En Afrique de l'Ouest, Macrotermes subhyalinus, Microtermes et Odontotermes sp. sont signalés comme nuisibles au manioc dans le Nord du Ghana (Maayiem et al., 2012). Au Bénin, Loko et al. $(2015 ; 2017)$ relèvent l'importance des dégâts causés par Amitermes evuncifer et Macrotermes subhyalinus sur l'igname, une plante à tubercule comme le manioc.

$\mathrm{Au}$ Malawi, en Afrique australe, Munthali et al. (1999) signalent sur le manioc des attaques de Macrotermes falciger, $M$. vitrialatus, Microtermes albopartitus, Microtermes lounsburyi et diverses autres espèces indéterminées de Microtermes et d'Odontotermes.

Les espèces du genre Microtermes et Ancistrotermes attaquent habituellement les tiges de l'intérieur à partir des racines et/ou du collet (Pearce 2006) causant ainsi des dommages importants. Chez l'igname par exemple, les pertes dues à ces deux genres peuvent dépasser 70\% (Rouland-Lefèvre, 2011).

Présente sur tous les sites et dans 18 parcelles $(\mathrm{F}=64 \%)$, Microtermes lepidus a la distribution la plus large à Tivaouane. Cependant, dans les travaux de Faye (2016) elle est qualifiée d'espèce accidentelle. La 
fréquence relativement faible de $M$. lepidus dans l'étude de Faye (2016), comparée à celle de Odontotermes erraticus, s'expliquerait par la méthode d'échantillonnage utilisée par cet auteur. En effet, Faye (2016) ne faisait pas de différence entre les termites rencontrés sur le manioc et ceux trouvés sur les arbres et autres résidus de cultures dans son aire d'échantillonnage.

La faible présence de Odontotermes erraticus sur le manioc observée, malgré sa forte présence dans les parcelles (placages sur ligneux, herbes et résidus de cultures), indiquerait sa faible appétence pour le manioc.

L'incidence moyenne des attaques sur le manioc observée $(37,5 \%)$ est comprise dans les valeurs notées par Atu (1993) sur le manioc au Nigéria (17 à 49\%). Faye (2016), à 60 jours après repiquage, a observé des incidences de 31 à $66 \%$ sur des variétés considérées comme sensibles et 1 à $19 \%$ sur d'autres qu'il qualifie de résistantes. L'étude montre également que l'incidence des attaques sur le manioc variait avec l'âge des plants. Elle est en moyenne de $53,4 \%$ pour les parcelles de 20 à 60 jours et de $28,4 \%$ pour les parcelles 90 à 425 jours. Cela est conforme aux observations d'Atu (1993) qui relève que les attaques du manioc par les termites se font principalement sur des boutures nouvellement implantées. Faye (2016) note également que les jeunes plants sont plus vulnérables aux attaques de termites.

L'indice de sévérité des attaques, $48 \%$ toutes parcelles confondues, est également plus élevée chez les jeunes plants $(72 \%$ pour une parcelle de 40 jours) que les plants âgés ( $1 \%$ pour une parcelle de 365 jours).

\section{Conclusion}

L'inventaire des espèces de termites qui attaquent le manioc cultivé dans le département de Tivaouane (région de Thiès) a permis de recenser les 5 espèces suivantes: Psammotermes hybostoma, Microcerotermes sp., Macrotermes subhyalinus, Microtermes lepidus et Odontotermes erraticus. Par leur constance dans les parcelles et leur fréquence sur le manioc élevées, Microtermes lepidus et Odontotermes erraticus sont des ravageurs importants du manioc à Tivaouane. Dans certaines parcelles des sites à sol sableux, Psammotermes hybostoma est responsable des attaques les plus sévères. Pour améliorer la productivité du manioc dans le département de Tivaouane, les termites devraient être pris en compte dans le développement de l'itinéraire technique du manioc.

\section{CONFLIT D'INTÉRÊTS}

Les auteurs reconnaissent l'absence de conflits d'intérêts entre eux.

\section{CONTRIBUTIONS DES AUTEURS}

AMS : définition du protocole, collecte des données sur le terrain, analyse statistique, rédaction du manuscrit. DS-S : définition du protocole, analyse statistique, rédaction manuscrit. $\mathrm{ABN}$ : coordination du travail, définition du protocole, identification des espèces, rédaction du manuscrit.

\section{REMERCIEMENTS}

Remerciements à M. Assane NDIAYE, Président de la Coopérative des Producteurs de Manioc du Département de Tivaouane (CODEPROMAT), pour son appui pendant l'échantillonnage des termites.

\section{REFERENCES}

Abderahim MS, Diatte M, Labou B, Sow G, Diarra K. 2018. Inventaire et distribution des principaux arthropodes ravageurs du manioc (Manihot esculenta CRANTZ) au Tchad. International Journal of Biological and Chemical Science, 12(6): 2589-2601.

DOI: https://dx.doi.org/10.4314/ijbcs.v12i6.10

Agre AP, Kouchade S, Odjo T, Dansi M, Nzobadila B, Assogba P, Dansi A, Akoegninou A, Sanni A. 2015. Diversité et évaluation participative des cultivars du manioc (Manihot esculenta Crantz) au Centre Bénin. Int. J. Biol. Chem. Sci., 9(1): 388-408. DOI : http://dx.doi.org/10.4314/ijbcs.v9i1.33.

Akpesse AA, Kouassi PK, Tano Y, Lepage M. 2008. Impact des termites dans les champs paysans de riz et de mais en savane sub-soudanienne (Booro- 
Borotou, Côte-d'Ivoire). Sciences \& Nature, 5(2): 121-131. DOI : http://dx.doi.org/10.4314/scinat.v5i2.421 58.

ANSD. 2015. Thiès. Situation économique et sociale régionale en 2013. Rapport ANSD, p. 139.

ANSD. 2017. Thiès. Situation économique et sociale régionale en 2014. Rapport ANSD, p. 157.

Atu UG. 1993. Cultural practices for the control of termite (Isoptera) damage to yams and cassava in south eastern Nigeria. International Journal of Pest Management, 39(4): 462-466. DOI: https://doi.org/10.1080/09670879309371 841.

Bouillon A, Mathot G. 1965. Quel est ce termite africain ? Zooleo, 1: 1-115.

Cacai GHT, Ahanhanzo C, Dangou JS, Houedjissin SS, Agbangla C. 2012. Effets de différentes combinaisons hormonales sur l'organogenèse in vitro de quelques cultivars locaux et variétés améliorées de Manihot esculenta Crantz (manioc-Euphorbiaceae) cultivées au Bénin. Int. J. Biol. Chem. Sci., 6(4): 1593-1607.

DOI http://dx.doi.org/10.4314/ijbcs.v6i4.19.

CDDR/SAILD. 2013. Lutte contre les maladies et ennemis du manioc (Manihot esculenta). CDDR/SAILD, p. 6.

Dajoz R. 1971. Précis d'écologie. Dunod: Paris.

DAPSA. 2013. Résultats prévisionnels de production agricole 2013/2014. Rapport DAPSA, p. 8.

DAPSA. 2017. Résultats définitifs de la campagne agricole, 2014-2015. DAPSA.

Diallo Y, Gueye MT, Sakho M, Gbaguidi DP, Kane A, Barthelemy JP, Lognay G. 2013. Importance nutritionnelle du manioc et perspectives pour l'alimentation de base au Sénégal (synthèse bibliographique). Biotechnol. Agron. Soc. Environ., 17(4): 634-643.

FAOSTAT. 2014. Statistiques production manioc Afrique de l'ouest. FAOSTAT. www.fao.org/faostat/fr (consulté le 17/02/2018).
Faye A. 2016. Etude comparative de variétés de manioc (Manihot esculenta CRANTZ) selon leur sensibilité aux termites ravageurs des boutures dans le département de Tivaouane (Sénégal) et leurs capacités organogénétiques in vitro. Thèse de Doctorat, Université Cheikh Anta Diop de Dakar, p. 160.

Faye A, Kane PD, Mbaye DF, Sall Sy D, Sané D. 2014. Study of the cassava varietal sensitivity to termites ravaging cuttings planted in farms in the department of Tivaouane (Senegal). International Journal of Science and Advanced Technology, 4(6): 6-16.

Grassé PP. 1944. Recherches sur la biologie des termites champignonnistes (Macrotermitinae). Annales des Sciences Naturelles, VI: 97-171.

Kotoklo EA, Kasseney BD, Nyamador W, Ketoh GK, Glitho AI. 2010. Attaques des arbres par les termites sur le campus de l'Université de Lomé (Togo). International Journal of Biological and Chemical Science, 4(1): 61-68. DOI: http://dx.doi.org/10.4314/ijbcs.v4i1.5423 1

Kotoklo AE, Amevoin K, Robert A, TanoY, Rouland-Lefevre C, Glitho IA. 2011. Degats causes par les termites sur les cannes à sucre au sud du Togo. Cameroon Journal of Biological and Biochimical Science, 19: 1-10. DOI: http://dx.doi.org/10.4314/ijbcs.v4i1.5423 1.

Kouakou J, Nanga Nanga S, Plagne-Ismail C, Aman MP, Ognakossan KE. 2015. Production et transformation du manioc. Collection Pro-Agro: YaoundéCameroun.

Loko YL, Agre P, Orobiyi A, Dossou-Aminon I, Roisin Y, Tamo M, Dansi A. 2015. Farmers' knowledge and perceptions of termites as pests of yam (Dioscorea spp.) in Central Benin. International Journal of Pest Management, 62(1): 7584.

DOI: 10.1080/09670874.2015.1107151.

Loko LYE, Orobiyi A, Agre P, Dansi A, Tamò M, Roisin Y. 2017. Farmers' 
perception of termites in agriculture production and their indigenous utilization in Northwest Benin. Journal of Ethnobiology and Ethnomedicine, 13: 64. DOI: 10.1186/s13002-017-0187-2.

Maayiem D, Bernard BN, Irunuoh AO. 2012. Indigenous knowledge of termite control: A case study of five farming communities in Gushegu District of Northern Ghana. Journal of Entomology and Nematology, 4(6): 58-64. DOI: 10.5897/JEN12.020.

Munthali DC, Logan JWM, Wood TG, Nyirenda GKC. 1999. Termite distribution and damage to crops on smallholder farms in southern Malawi. International Journal of Tropical Insect Science, 19(1): 43-49. DOI: https://doi.org/10.1017/S1742758400016 $57 \mathrm{X}$.

Ndiaye AB, Han SH. 2002. Attaque des arbres fruitiers par les termites en Casamance (Sénégal) (Isoptera). Bulletin de la société entomologique de France, 107 (2) : 193-199.

Ndiaye AB, Han SH. 2006. L'attaque des arbres fruitiers par les termites dans la région de Thiès (Sénégal) (Isoptera). Bulletin de la société entomologique de France, 111(1): 59-64.

Ndiaye AB, Han SH. 2007. Les termites (Isoptera) des arbres fruitiers de la région de Kaolack (Sénégal). Bulletin de l'Institut fondamental d'Afrique noire série A, 52(1-2): 147-161.
Ndiaye AB. 2014. Contribution à la connaissance des termites (Isoptera Brullé, 1832) du Sénégal : Systématique et Ecologie. Thèse de doctorat èssciences, Université Cheikh Anta Diop de Dakar, Faculté des Sciences et Techniques, p. 257.

Pearce MJ. 2006. Termites: Biology and pest management. $\mathrm{CAB}$ International: Wallingford.

Sané CAB, Rouland-Lefèvre C, Grechi I, Rey JY, Vayssieres JF, Diamé L, Diarra K. 2016. Diversité, nuisances et modes de gestion des termites (Isoptera) dans les agrosystèmes sénégalais. Int. J. Biol. Chem. Sci., 10(1): 134-154. DOI: http://dx.doi.org/10.4314/ijbcs.v10i1.10.

Sonko AM. 2015. Les attaques des termites (Isoptera, Brullé) sur le manguier (Mangifera indica L.) dans les vergers du plateau de Diass et des Niayes de Bayakh (Sénégal). Mémoire de master II, Université Ch. Anta Diop, Dakar, p. 49.

SCA. 2013. Etude sur l'organisation et la structuration de filières agricoles au Sénégal en des chaînes de valeur performantes dans le cadre de la Grappe « Agriculture et Agro-industrie ». Chaine de valeur manioc, rapport SCA, p. 20.

Vernier P, N'Zué B, Zakhia-Rozis N. 2018. Le manioc, entre culture alimentaire et filière agro-industrielle. Agricultures tropicales en poche, Editions Quae: Versailles. 